\title{
Serum calcitonin gene related peptide (CGRP) levels in migraine: A study on its clinical correlation and diagnostic efficacy
}

\author{
Sofia Khatoon ${ }^{1}$, Noorunissa Begum 1 , Hafeeza Sultana', Maryam Rashed', Mohammed Zoheb², \\ Aleem Ahmed Khan ${ }^{3}$, Sara Shreen ${ }^{4}$, Syed Iqbal Ahmed ${ }^{4}$
}

\author{
${ }^{1}$ Department of Pharmacy Practice, Deccan School of Pharmacy, Hyderabad, India \\ ${ }^{2}$ Department of Neurology, Owaisi Hospital and Research Center, Deccan College of Medical Sciences, Hyderabad, India \\ ${ }^{3}$ Central Research lab, Center for Liver Research and Diagnostics, Deccan College of Medical Sciences, Hyderabad, India \\ ${ }^{4}$ Department of Pharmacy practice, Deccan School of Pharmacy, Hyderabad, India
}

\begin{abstract}
Migraine is a primary headache disorder marked by recurrent unilateral headache episodes. Calcitonin gene related peptide (CGRP) plays major role in migraine pathophysiology. CGRP is multifunctional, and its vasodilating activity within the central and peripheral blood vessels is one in all its primary functions.The intention is to prove serum calcitonin gene related peptide (CGRP) as an early diagnostic tool for migraine and the novelty is to correlate it with characteristics of migraine so that it helps in early initiation of treatment.

Methods. 100 subjects including 90 patients with migraine and 10 with non-headache (NH) age-matched controls were prospectively recruited in our current study. The subjects were aged from $15-50$ years. The clinical assessment was made every month for the three months after the start of therapy. The subjects were compared based on the serum CGRP values. Serum CGRP concentrations were measured by using CGRP ELISA kit.

Results. Out of total subjects selected, the maximum (23.3\%) subjects were between age 26 and 30 years and least effected age group was $46-50$ years (4.44\%). Females' predominance with $82 \%$ than males with $18 \%$.Stress was major trigger occurring in 57\% of cases. Throbbing pain with elevated CGRP levels $130.44 \pm 114.22$ and $p$ value $(p=0.01)$. The average CGRP levels was higher in test group $149.00 \pm 93.86$ compared to control $61.30 \pm 24.37$ with $p$ value $(p=0.02)$.

Conclusions. The serum CGRP levels were statistically more in migraine patients correlated with characteristics like throbbing type of pain, stress and inadequate sleep. Hence, the serum CGRP levels estimation can be considered as a diagnostic tool for migraine when the clinical character's over lap or early in the course of migraine when all criteria for diagnosis are not yet fulfilled.
\end{abstract}

Keywords: migraine, CGRP, neuralgia, ICHD, photophobia, phonophobia, ELISA

\section{INTRODUCTION}

Migraine is a primary headache disorder marked by unilateral recurrent headache episodes with various proposed patho-physiological mechanisms. Migraine can be with or without aura in its mild form may have nausea, giddiness and inflated sensitivity to light and sound (1). Headaches presents as epi- sodic attacks of variable frequency that disturbs the conventional daily activities , taking a toll on physical, mental and social well being and has severe economic consequences (2).

There are triggers that increase the frequency or severity of headache like stress and anxiety, alkaloid or alcohol intake, inadequate sleep, inadequate 
food, environmental changes, changes in women throughout menstrual cycle, food that contains nitrates (meats, hot dogs), aminoalkanoic acid (cheese, smoke-cured fish), flavouring, sweetener (3).

The identification of headache is supported by International Headache Society Criteria. The two main sorts of migraine are migraine without aura and migraine with aura.

Migraine without aura is termed as common headache. A. Five attacks fulfilling criteria B-D. B. Headache attacks lasting 4-72 60 minutes (untreated or unsuccessfully treated). C. Headache has 2 of the subsequent four characteristics: unilateral location, beating quality, moderate or severe pain intensity, aggravation by or inflicting rejection of routine physical activity (e.g.,walking or ascension stairs). D.Throughout headache one in all the following: Nausea and/or instinctive reflex, photophobia and acousticophobia E. Not higher accounted for by another ICHD-3 identification.

Migraine with aura is termed as classic headache. A. Two attacks fulfilling criteria B. B. One or a lot of of the subsequent absolutely reversible aura symptoms: Visual sensory, speech and/or language, motor, brainstem, retinal. C. Three of the below characteristics: one aura symptom spreads step by step over $\geq 5$ minutes; two or a lot of aura symptoms occur in succession; every individual aura symptom lasts 5-60 minutes; one aura symptom is unilateral; one aura symptom is positive; the aura is accompanied, within 60 minutes by headache (4).

CGRP is 37 amino acid neuropeptide (5). It has two forms, aCGRP and $\beta$-CGRP.aCGRP is present across the central and peripheral nervous system. $\beta$-CGRP is positioned within the enteric system and therefore has the endocrine role (6). CGRP is multifunctional, and its vasodilating activity within the central and peripheral blood vessels is one in all its primary functions (5). CGRP has several essential physiological functions (7). The CGRP receptor, as characterised by IUPHAR, is a convolution between thyrocalcitonin receptor-like receptor (CLR) and receptor activity-modifying supermolecule one (RAMP1). It is the primary receptor to be recognized as a convolution between a $\mathrm{G}$ supermolecule coupled receptor (GPCR) and a RAMP. Various mechanisms are proposed to be involved in the release of CGRP in migraine from the trigeminal ganglia. The most accepted theory is of a state of neuroinflammation.

\section{AIM}

Hence the aim of this study was to estimate the serum CGRP levels in migraine patients and the objective is to correlate the levels with certain characteristics of migraine.CGRP levels estimation could facilitate in assessing its role as diagnostic tool and if the serum CGRP level shows correlation with the characteristics, it should facilitate in early diagnosis which in turn helps in initiation of treatment. The patients are enrolled based on ICHD diagnostic criteria, taking under consideration the patient's complete history so as to grasp details on previous medications or diseases which may be the reason for headache.

\section{MATERIALS AND METHODS}

Our study is a prospective and observational study with sample size 100 subjects with study duration of six months conducted at neurology out-patient department and Central Research Laboratory, Owaisi Hospital and Research Center, Hyderabad.

\section{Ethical approval}

This study has been approved by Institutional review board (IRB), on 27 November, 2019 at Deccan College of Medical Sciences and Allied Hospital, Hyderabad, Telangana.

\section{Study criteria}

Inclusion criteria: patients presenting with migraineous headache, patients ensuing ICHS guidelines, newly diagnosed episodic migraine patients, patients above 18 \& less than 50 years of age.

Exclusion criteria: subjects unwilling to participate in the study, pregnant women, patient with comorbidities seizures, CVA, stroke.

\section{Study procedure}

We prospectively recruited 100 adult patients with 90 migraineurs and 10 normal controls at Owaisi Hospital and research center from August 2019 to April 2020. The study subjects were aged from 15 to 50 years. We assessed patients at the outpatient services of Neurology department. A detailed history was taken to assess the type, pattern, severity and duration of headache. Those matching our study criteria were included. Patients presenting with episodic migraine were included. Steps were taken to avoid including those individuals who have overlap features with other types of headaches, those who have taken any prophylactic medication or other medications so as to strengthen the quality of study and its analysis. The diagnosis of migraine was based on the International classification of headache disorder $3^{\text {rd }}$ edition beta version (ICHD-3beta). After detailed history taking and examination, patients satisfying the criteria of study were selected and an informed consent was taken. 4 $\mathrm{ml}$ blood samples were collected within 24 hours of the headache attack and CGRP levels were estimat- 
ed by using commercially available ELISA kit with quantitave analysis. Follow-up of patients was done either on phone or with a personal visit to opd. Perceived stress scale was used for assessment of stress and headache was subjectively assessed.

\section{Statistical analysis}

Statistical analysis was performed using SPSS software (version 20.0). Unpaired t test was used to find out the significant differences between two groups which were illustrated as mean and standard deviation. $t$ is the test statistics that measures the statistical relationship or association between two continuous variables. It gives information about the magnitude of the association, or correlation as well as the direction of the relationship. Multivariate analysis was included to consider correlation between various factors.

\section{RESULTS}

\section{Age distribution}

Out of 90 subjects selected, $23.3 \%$ patients between age 26 and 30 years are affected with migraine irrespective of gender, followed by age group 21-25 years with $23.3 \%$ and age group 46-50 years with least $4.44 \%$ of patients affected. Mean age is (mean \pm SD) $30.51 \pm 8.64$.
TABLE 1. Age distribution

\begin{tabular}{|c|c|c|}
\hline Age groups & Number of subjects & Percentage \\
\hline $15-20$ & 13 & 14.4 \\
\hline $21-25$ & 17 & 18.8 \\
\hline $26-30$ & 21 & 23.3 \\
\hline $31-35$ & 16 & 17.7 \\
\hline $36-40$ & 12 & 13.3 \\
\hline $41-45$ & 7 & 7.77 \\
\hline $46-50$ & 4 & 4.44 \\
\hline
\end{tabular}

\section{Gender distribution}

Among all the subjects there was female predominance with $82 \%$ compared to male subjects with $18 \%$.

\section{Triggers causing migraine}

Among all the subjects, stress was found to be the major trigger in migraine patients occurring in $57 \%$ of cases, while workload was only $12 \%$ trigger in subjects.

In table 2, we compared the CGRP levels with clinical manifestations. It was observed that patients with throbbing pain were found with high CGRP levels $130.44 \pm 114.22$ compared to that of absence of throbbing pain.88.37 \pm 73.42 .The $p$ value was found to be significant (i.e. $p=0.01$ ).
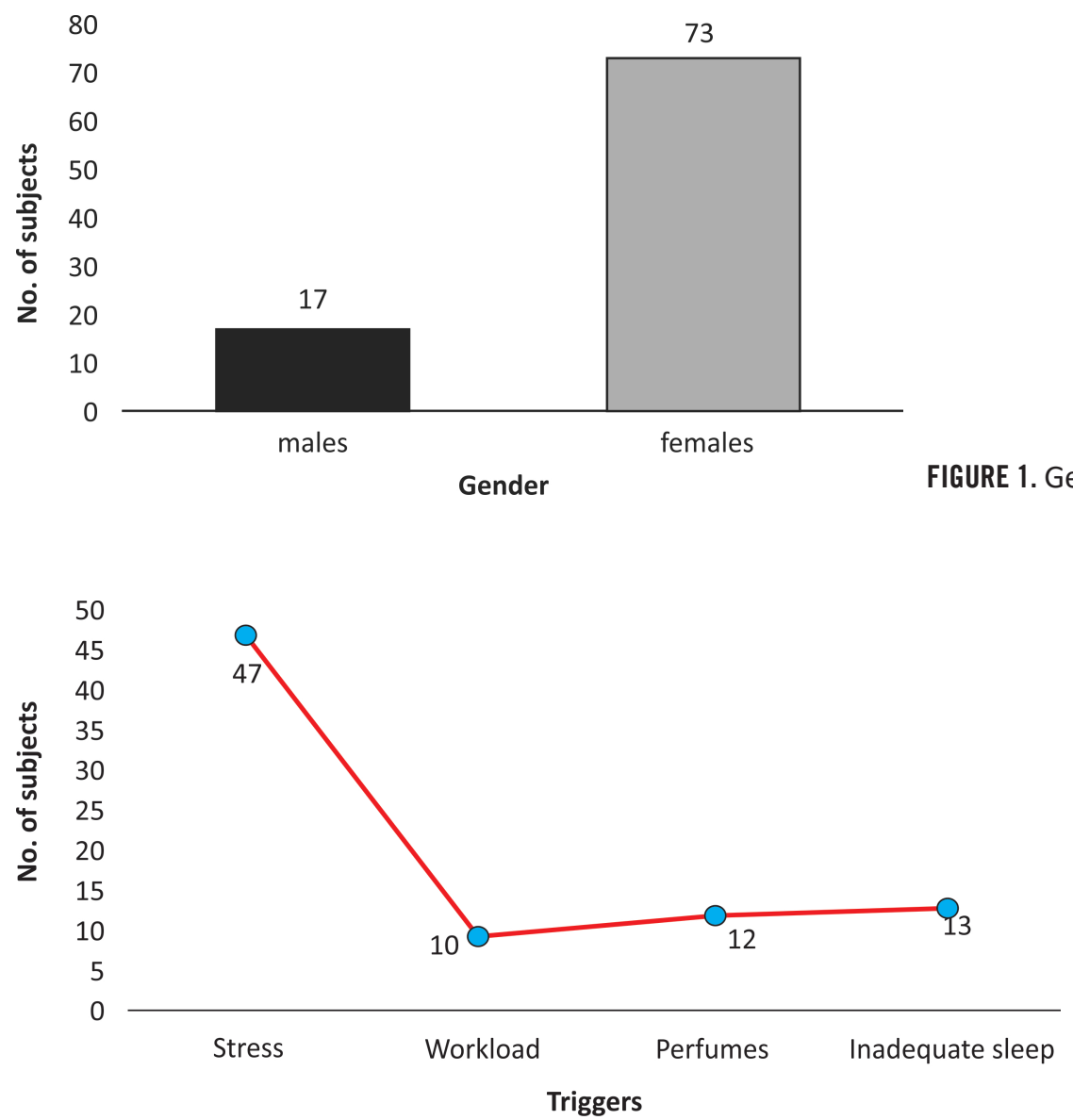

FIGURE 1. Gender distribution in study subjects 
TABLE 2. Details of association between CGRP levels and clinical manifestations

\begin{tabular}{|l|c|c|c|c|c|c|}
\hline Variables & Positive & $\begin{array}{c}\text { Mean \& SD } \\
\text { value }\end{array}$ & Negative & $\begin{array}{c}\text { Mean \& SD } \\
\text { value }\end{array}$ & t & $\begin{array}{c}\text { p } \\
\text { value }\end{array}$ \\
\hline Type & \multicolumn{7}{|l|}{\begin{tabular}{l} 
tue \\
\hline Throbbing
\end{tabular}} & 44 & $\mathbf{1 3 0 . 4 4 \pm 1 1 4 . 2 2}$ & 46 & $88.37 \pm 73.42$ & 2.55 & $\mathbf{0 . 0 1 *}$ \\
\hline Frequent & 68 & $151.05 \pm 99.61$ & 22 & $114.62 \pm 109.99$ & 1.19 & 0.24 \\
\hline Trigger & 48 & $137.05 \pm 86.34$ & 42 & $86.07 \pm 53.62$ & 2.26 & $\mathbf{0 . 0 3 *}$ \\
\hline Stress & 11 & $120.60 \pm 111.77$ & 79 & $127.50 \pm 81.05$ & 0.14 & 0.89 \\
\hline Workload & 11 & $102.50 \pm 82.58$ & 78 & $135.25 \pm 100.69$ & 0.80 & 0.43 \\
\hline Perfumes & 13 & $108.77 \pm 79.31$ & 76 & $192.23 \pm 102.34$ & 1.90 & $\mathbf{0 . 0 5 *}$ \\
\hline Inadequate sleep & 21 & $121.57 \pm 110.48$ & 68 & $110.48 \pm 48.63$ & 0.12 & 0.72 \\
\hline Location & 53 & $135 \pm 87 \pm 127.62$ & 36 & $127.62 \pm 58.36$ & 0.87 & 0.42 \\
\hline Unilateral & 47 & $122.68 \pm 62.38$ & 42 & $108.58 \pm 42.89$ & 0.82 & 0.19 \\
\hline Bilateral &
\end{tabular}

Moreover, CGRP levels were observed to be elevated in patients with stress $137.05 \pm 86.34$ than patients without stress $86.07 \pm 53.62$. The $\mathrm{p}$ value was found to be significant (i.e. $\mathrm{p}=0.03$ ).

Similarly, CGRP levels were increased in patients with inadequate sleep $192.23 \pm 102.34$ than the one's with adequate sleep $108.77 \pm 79.31$. The difference was statistically significant $(p=0.05)$.

\section{The comparison of CGRP between test and control subjects}

The average CGRP levels in test group were $149.00 \pm 93.86$ whereas in control it was $61.30 \pm 24.37$. The difference was statistically significant $(p=0.02)$.

Multivariate regression analysis (showed in figure $3 \mathrm{~A}, \mathrm{~B}, \mathrm{C})$ is suggestive of significant correlation between factors like throbbing type of pain, stress, inadequate sleep, sensitivity to perfumes and pattern of headache with higher CGRP values.

\section{DISCUSSION}

There is no effective diagnosing tool for chronic and acute migraine, CGRP levels can help in differentiating migraine and other types of headache. A state of neuroinflammation is thought to precipitate the release CGRP which in turn leads to vasodilatation causing headache. However, many hypothesis are proposed as precipitating factors for inflammation but none have been proven yet. As the previous studies proved that the CGRP plays an integral role in migraine pathophysiology as its levels increases in migraine $(4,8,9)$, estimating CGRP in the serum may help in early diagnosis of migraine. Previous studies have also proved plasma CGRP as a potential biomarker for migraine $(1,5)$, but in our present study we have taken serum CGRP levels of adult migraine patients into consideration. The prediction of serum CGRP levels and the novel idea of correlat- ing it with the migraine characteristics is the core purpose of our study.

We have enrolled 100 subjects in our study, which are divided as 90 migraine patients and 10 normal controls (without headache or migraine symptoms). The selection of subjects was based on ICHD 3 beta guidelines, drug therapy was given to the patients and clinical assessment was done every month for about three months. The blood samples were collected and the centrifuged for separation of serum from blood and serum CGRP levels were estimated by using commercially available CGRP ELISA kit.

Among total of 90 subjects, 17 were male and 73 were female patients, indicating female predominance, consistent with previous studies. It is thought that probably hormonal fluctuations and varied receptor sensitivity in females is responsible for more female preponderance.Similarly if there is a difference of CGRP sensitivity and levels between either sexes, has to be assessed in a separate study. Majority of patients i.e.,23.3\% affected with migraine were in between age 26 and 30 years and age group 45-50 years was the least effected i.e. $4 \%$. It has been reported by Leslie Kelman that there is age related decline in triggers of migraine like stress, photophobia, phonophobia throbbing and pressure due to headache (10). CGRP is thought to exert its vasodilating effect through nitric oxide (NO) and also an antiproliferative effect. As age progresses the levels of NO decrease which might to an extent explain the age related variation of CGRP, which inturn explains reduction in migraine. The estimated CGRP values were compared for different parameters like symptoms, triggers and treatment response.No studies have been published to compare the CGRP levels in relation to various triggers and clinical features.

The comparison of CGRP levels with clinical manifestations revealed the statistical significance, as patients with throbbing pain were found with el- 


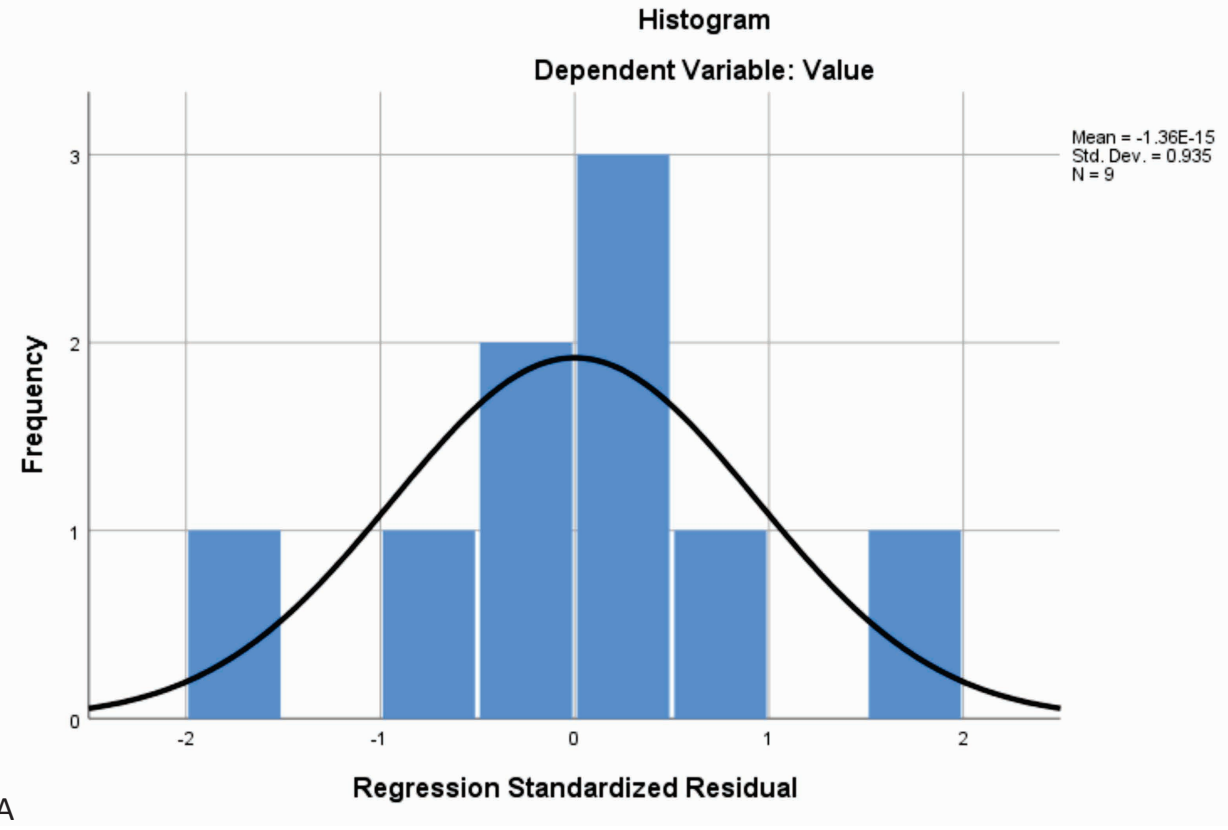

Normal P-P Plot of Regression Standardized Residual

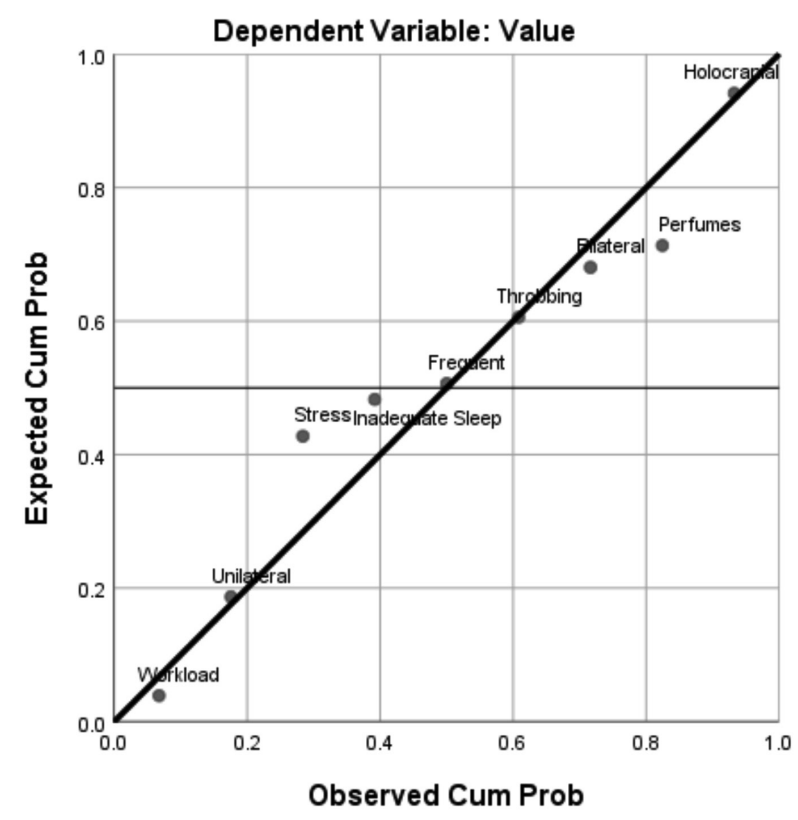

B

Scatterplot

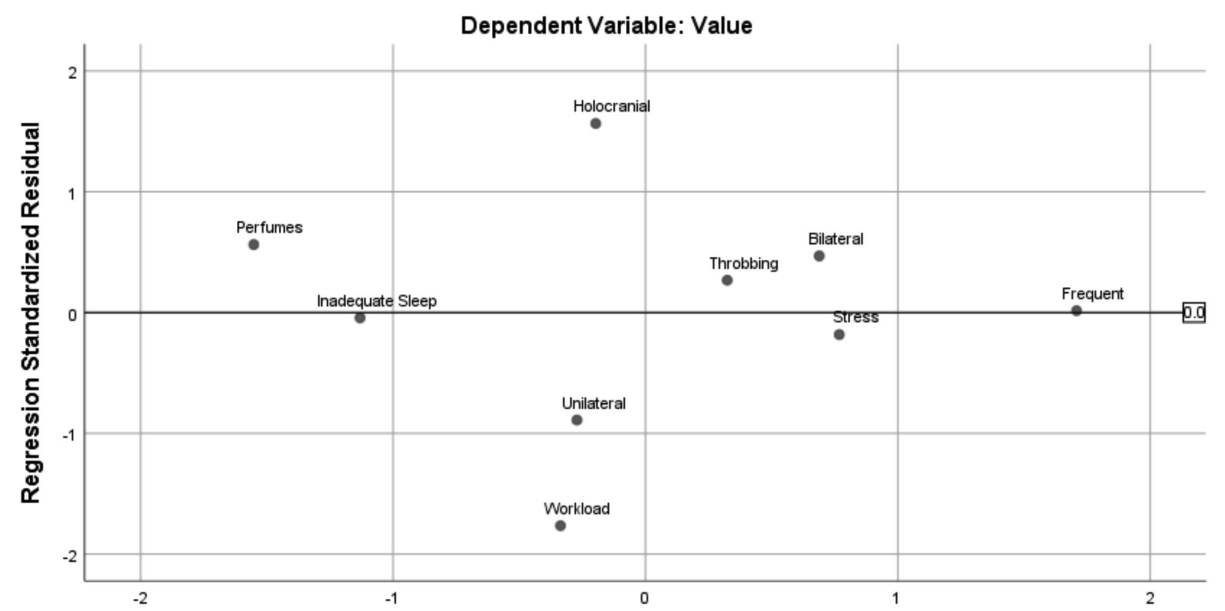

C 
evated CGRP levels $130.44 \pm 114.22$ with p value 0.01 . A study conducted by Chou et al. concluded that an increase in intracranial pressure is responsible for initiating throbbing pain, also low intracranial pressure can in some cases accentuate throbbing pain (11). Moreover, CGRP levels were increased in patients with triggers like stress with high PSS score $137.05 \pm 86.34$ where as other patients without stress or low PSS score $86.07 \pm 53.62, p=0.03$. Stress is associated with various hormonal changes and also elevation of inflammatory markers. These factors might inturn elevate the CGRP levels. Similarly, CGRP levels were increased in patients with inadequate sleep $192.23 \pm 102.34$ than patients with adequate sleep 108.77 $\pm 79.31(\mathrm{p}=0.05)$. This shows the statistical significance for stress and inadequate sleep which indicates that stress along with sleep deprivation are the high-risk factors for migraine. Stress ratings, duration of previous nights' sleep, and headache severity were evaluated using a series of linear mixed models with random effects in a study conducted by Houle et al. to observe individual differences. High stress and inadequate sleep are major risk factors for predicting the severity of headache (12).

The average CGRP levels in test group were $149.00 \pm 93.86$, which were higher than the controls, it was $61.30 \pm 24.37$. The difference was statistically significant $(p=0.02)$. Previous studies have shown doubtful role of CGRP as a biomarker or diagnostic tool in migraine.In our study a statistically significant value signifies its role as a diagnostic tool how ever we propose its role depends on the phase of headache during which it is assessed .CGRP levels in adult patients are higher than those in healthy controls at the baseline (13). During migraine attacks, plasma CGRP levels are elevated in adults (13) and their changes shows relation with headache intensities $(5,14)$.

Hence, serum CGRP levels were statistically correlated with characteristics like throbbing pain, stress and inadequate sleep. Which means that throbbing pain, inadequate sleep along with stress are the risk factors for migraine. Patients were either prescribed monotherapy or dual therapy after evaluation of symptoms on the very first visit to the hospital.

Drugs used in mono therapy: Naproxen $500 \mathrm{mg}$ (3-5 days) along with Sibelium or Flunarizine 5/10 mg (30 days). Whereas, the dual therapy includes Naproxen 500 mg (35 days) along with Flunarizine 5 $\mathrm{mg} / 10 \mathrm{mg}$ (30 days) and Tab. Inderal la (Propranolol) 40 mg-1 month (or) Tab. Topamac (Topiramate) 25 mg-1 week (or) Tab. Amicon (Amitryptiline) 10 mg-1 month. The patients were assessed clinically every month for three months. In patients who showed no response to monotherapy, the drug therapy was changed to dual.

Like all studies, there are some limitations in this study too. Firstly, the patient's volume is less (i.e., 100). Large sample size will provide more details about the statistical parameter. Secondly, a larger study with measurement of serum CGRP at baseline and ictal or interictal values may provide more information to include CGRP as biomarker in diagnostic criteria in future.

Till date, no studies have been done to predict the correlation between serum CGRP levels and migraine characteristics hence we provide the data and correlation between various factors.

\section{CONCLUSIONS}

From our study we concluded the serum CGRP levels are elevated in migraineurs. Patients with high stress, inadequate sleep and throbbing pain had significantly higher CGRP levels when compared to the control group, indicating that these are the major risk factors in migraine. Thus, serum CGRP levels estimation by ELISA can be used as a diagnostic tool for migraine which will help in early start of treatment.

\section{Acknowledgement}

It is a pleasure task to express our thanks to all those who contributed in many ways to the success of this study. It gives us immense pleasure to acknowledge our heartfelt gratitude to all who rendered their kind help in preparing this dissertation. The valuable suggestions, affectionate encouragement, generous help and important acumen of all co-authors are greatly acknowledged. We should be failing in our duties if we don't mention the help of authors of journals and books, the pillars of our research work.

Conflict of interest: none declared Financial support: none declared

\section{REFERENCES}

1. Puledda F, Messina R, Goadsby PJ. An update on migraine: current understanding and future directions. J Neurol. 2017 Sep; 264(9):2031-2039.

2. de Prado BM, Russo AF. CGRP receptor antagonists: A new frontier of anti-migraine medications. Drug discovery today. Therapeutic Strategies. 2006;3(4):593-597.

3. Hildreth CJ, Lynm C, Glass RM. Migraine Headache. JAMA. 2009; 301(24):2608.

4. Guidelines [Internet]. International Headache Society. Available at: https://ihs-headache.org/en/resources/guidelines/.

5. Fan PC, Kuo PH, Lee MT, Chang SH, Chiou LC. Plasma Calcitonin Gene-Related Peptide: A Potential Biomarker for Diagnosis and 
Therapeutic Responses in Pediatric Migraine. Front Neurol. 2019 Jan 24;10:10.

6. Villalón CM, Olesen J. The role of CGRP in the pathophysiology of migraine and efficacy of CGRP receptor antagonists as acute antimigraine drugs. Pharmacol Ther. 2009 Dec;124(3):309-23.

7. Durham PL, Vause CV. Calcitonin gene-related peptide (CGRP) receptor antagonists in the treatment of migraine. CNS Drugs. 2010 Jul;24(7):539-48.

8. Cernuda-Morollón E, Larrosa D, Ramón C, Vega J, Martínez-Camblor P, Pascual J. Interictal increase of CGRP levels in peripheral blood as a biomarker for chronic migraine. Neurology. 2013 Oct 1;81(14):1191-6.

9. Mulderry PK, Ghatei MA, Spokes RA, Jones PM, Pierson AM, et al. Differential expression of alpha-CGRP and beta-CGRP by primary sensory neurons and enteric autonomic neurons of the rat. Neuroscience. 1988 Apr;25(1):195-205.
10. Kelman L. Migraine changes with age: IMPACT on migraine classification. Headache. 2006 Jul-Aug;46(7):1161-71.

11. Chou $\mathrm{CH}$, Fuh JL, Hu HH, Wu JC, Wang SJ. Throbbing pain is related to Queckenstedt's test effect in migraine patients. Cephalalgia. 2009;29(3):373-378.

12. Houle TT, Butschek RA, Turner DP, Smitherman TA, Rains JC, Penzien DB. Stress and sleep duration predict headache severity in chronic headache sufferers. Pain. 2012 Dec;153(12):2432-2440.

13. Juhasz G, Zsombok T, Modos EA, Olajos S, Jakab B, Nemeth J, et al. NO-induced migraine attack: strong increase in plasma calcitonin gene-related peptide (CGRP) concentration and negative correlation with platelet serotonin release. Pain. 2003;106:461-70.

14. Goadsby PJ, Lipton RB, Ferrari MD. Migraine-current understanding and treatment. N Engl J Med. 2002;346:257-70. 